\title{
Análisis comparativo del discurso sobre Innovación Disruptiva en los medios de comunicación y la literatura académica
}

\author{
Daniela Buzova ${ }^{a}$, Silvia Sanz-Blas ${ }^{\text {b }}$, Cristina Santos-Rojo ${ }^{c}$, Agustín Carrilero-Castillo ${ }^{d}$ \\ ${ }^{a}$ ESIC Business \& Marketing School, Valencia, España, daniela.buzova@esic.edu, ${ }^{b}$ Departamento de \\ Comercialización e Investigación de Mercados, Universitat de Valencia, España, silvia.sanz@uv.es, \\ ${ }^{c}$ ESIC Business \& Marketing School, Valencia, España, cristina.santos@esic.edu, ${ }^{\mathrm{d}}$ ESIC Business \& \\ Marketing School, Valencia, España, agustin.carrilero@esic.edu
}

\section{Resumen}

El presente trabajo tiene por objetivo comparar el discurso sobre el fenómeno de innovación disruptiva en los medios de comunicación con los temas tratados por la investigación académica en este ámbito. Para ello, se ha consultado la base de datos de prensa internacional Factiva, de la cual se han recopilado 865 noticias relacionadas con el tema. Por otro lado, la búsqueda del término "innovación disruptiva” en la Colección Principal de la base de datos ISI Web of Science dio como resultado 161 artículos académicos publicados en el periodo 2004-2019. Para el análisis de contenido se utilizará el programa de minería de texto Leximancer. El estudio pretende contribuir a la literatura analizando cómo los medios de comunicación, en su rol de dinamizadores del debate público y facilitadores de la aceptación de las innovaciones disruptivas describen este fenómeno y si están alineados con los avances en la investigación académica.

Palabras clave: Innovación disruptiva, medios de comunicación, revisión de literatura, análisis de contenido, Leximancer.

\section{Abstract}

This study aims to compare the media discourse on the disruptive innovation phenomenon with the academic literature published on that topic. For this purpose, 864 news articles referring to disruptive innovation were retrieved from the international news database Factiva. In addition, 161 academic papers including the term "disruptive innovation" were found on the Core collection of Web of Science published from 2004 until 2019. The automatic content analysis of the data will be carried out with the text-mining software Leximancer. The expected contribution of the study is related to uncovering 
how the media, in its role of agenda-setter and facilitator of the adoption of disruptive innovations describe this phenomenon and whether its discourse is aligned with the advancements in the academic research.

Keywords: Disruptive innovation, media communication, literature review, content analysis, Leximancer.

\section{Introducción}

La innovación disruptiva es un fenómeno que ha irrumpido con fuerza en la economía global y como tal ha sido objeto de interés no solo del ámbito empresarial, sino también de la comunidad académica, y la sociedad, en general. Gracias al desarrollo de las tecnologías de la información y la comunicación (TIC), los procesos de innovación disruptiva han tenido un impacto relevante en muchos sectores, permitiendo la accesibilidad a una serie de productos y servicios que de otra forma solo estarían al alcance de los consumidores con un elevado poder adquisitivo (Christensen et al., 2015). Sin embargo, a pesar de los beneficios netos que proporciona la innovación disruptiva, este fenómeno no siempre ha sido recibido positivamente por los mercados, ya que, al redefinir la trayectoria del funcionamiento de un producto o servicio, las empresas dominantes se pueden ver perjudicadas (Gilbert, 2003; Sandström et al., 2009).

Estudios previos señalan que los medios de comunicación juegan un papel relevante en la aceptación de las transformaciones ocasionados por la introducción de innovaciones disruptivas en los mercados (Laurell y Sandström, 2018). El discurso que adoptan los medios de comunicación puede afectar el ritmo de aceptación de las innovaciones, debido a su función de no solo informativa, sino también dinamizadora del debate social sobre los temas tratados (Gómez, 2009; Jensen, 2002).

No obstante, hasta la actualidad no se ha encontrado ningún estudio que analice la configuración de la agenda mediática alrededor del fenómeno de innovación disruptiva y compare dicho discurso con los temas tratados en la investigación científica sobre este concepto. Así el objetivo principal del trabajo se centra en determinar si las dos áreas están alineadas y en caso contrario detectar aquellos temas de investigación que necesitan mayor diseminación mediática o aquellos temas en la agenda mediática que no han sido tratados por los estudios científicos hasta el momento. 


\section{Marco teórico}

El término "innovación disruptiva" fue acuñado por Christensen en el año 1997 para designar el proceso en el cual se logra transformar un producto o servicio que es sofisticado y/o caro, en uno más simple y/o económico, con la finalidad de crear nuevos mercados y democratizar su uso (Christensen et al., 2015; Hopp et al., 2018). La innovación disruptiva ha sido objeto de estudio de numerosas investigaciones hasta la actualidad (Shang et al., 2019) que han examinado este fenómeno desde diferentes perspectivas entre los cuales destaca la innovación arraigada en la tecnología (Li et al., 2018), la innovación como proceso (Petzhold et al., 2019) o la innovación disruptiva como modelo de negocio (Zhang et al., 2018).

Desde su creación, la teoría de la innovación disruptiva ha sido sometida a discusiones por parte de varios autores (Markides, 2006; King y Baatartogtokh, 2015; Petzhold et al., 2019), pero la mayoría coincide en que se trata de un proceso de innovación que ocasiona una ruptura con la forma en la que se comercializaba un producto o servicio, llegando incluso a llevar ese producto o servicio a sobrepasar en aceptación a la oferta ya implantada en el mercado (Schmidt et al., 2008). Un ejemplo ilustrativo, que ha suscitado mucha polémica recientemente, es el sector turístico con el crecimiento de plataformas peer-topeer como Airbnb (Dogru et al., 2019). El subsector hotelero ha visto cómo sus reglas de juego han cambiado a raíz de la "democratización" del alojamiento turístico, lo que ha tenido un eco importante en los medios de comunicación (Leung et al., 2019).

Dado el efecto transformador de la introducción de innovaciones disruptiva en los mercados, el papel de los medios de comunicación como catalizadores de la aceptación de estos procesos ha sido destacado por varios autores (Laurell y Sandström, 2018; Martin, 2016). Los medios de comunicación influyen en las opiniones de los consumidores (DjerfPierre y Shehata, 2017), al cumplir la función de "agenda-setter" (McCombs, 2005), que refleja el poder de atraer la atención hacia ciertos temas, que a su vez determinan la agenda social. En esta relación, recientemente, han surgido estudios que examinan la cobertura mediática de diversos tipos de innovación disruptiva con el fin de saber cuál es el contexto mediático en el cual se inscriben estos nuevos productos/servicios o modelos de negocios. Por ejemplo, varios autores se han centrado en determinar el discurso mediático sobre la economía colaborativa y su impacto en el sector hotelero y el del transporte (Cheng y Edwards, 2019; Laurell y Sandström, 2018). 


\section{Metodología}

\subsection{Recogida de datos}

La recogida de datos se realizó acudiendo a dos bases de datos distintas. Por un lado, para la recogida de artículos académicos relacionados con la temática de innovación disruptiva se eligió la base de datos ISI Web of Science, por ser considerada como la plataforma que alberga el conocimiento científico con mayor factor de impacto en la ciencia (Falagas et al., 2008). Más concretamente, la búsqueda de artículos académicos se realizó en la colección principal de la base de datos introduciendo el término de búsqueda "disruptive innovation" como tema. Para delimitar los resultados de la búsqueda se filtró por temática para así descartar aquellos trabajos que utilizaban el término en otros contextos irrelevantes para el estudio como por ejemplo medicina, ingeniería o educación. De esta forma, finalmente se obtuvo un total de 161 artículos académicos publicados en el periodo 2002 a 2019.

Por lo que respecta a las noticias publicadas sobre innovación disruptiva se utilizó la base de datos documental Factiva, que ofrece acceso a una colección de más de 32.000 periódicos y revistas de 150 países. La búsqueda del término "innovación disruptiva" en inglés dio como resultado 2823 noticias publicadas desde la primera vez que aparece la palabra en el año 2008 hasta finales de 2019. Sin embargo, igual que en el caso anterior no todas las noticias son relevantes para el caso de estudio. Por ello, se ha filtrado por temática, que Factiva proporciona de forma automática, para incluir solo aquellas noticias incluidas en la categoría "Innovación empresarial/Disruptiva". Después de eliminar las duplicidades, la muestra final asciende a 865 noticias. Para cada documento encontrado se obtuvo el título de la noticia, la fecha de publicación, el texto completo y el medio donde fue publicado.

\subsection{Análisis de datos}

Para llevar a cabo el análisis de contenido de los 161 artículos académicos y las 864 noticias de prensa se utilizará el programa de minería de texto Leximancer. El método que utiliza el software permite "transformar la información de co-ocurrencias en el lenguaje natural en patrones semánticos de una forma automática sin necesidad de supervisión (Smith y Humphreys, 2006, p. 262). Este software cualitativo se basa en algoritmos semánticos que primero "aprenden" categorías de conceptos del corpus, después codifican los segmentos de texto en función de las categorías y, por último, analizan las relaciones entre los conceptos identificados. 
Los datos extraídos se muestran a través de mapas conceptuales que proporcionan una panorámica general del contenido textual. Los mapas reflejan no solo los conceptos más relevantes que configuran el texto, sino también cómo éstos se relacionan entre sí. Cada tema se representa a través de un círculo de un determinado color, que contiene varios conceptos interrelacionados, siendo el círculo más grande y de color más claro el más relevante. La distancia entre los diferentes temas y conceptos en el mapa indica el grado de relación entre ellos, esto es, los conceptos que se muestran más cerca unos de otros se encuentran también más frecuentemente juntos en el texto. Además del mapa general de temas, Leximancer también permite perfilar los datos por categorías de interés como, por ejemplo, el año de publicación, el género o el grado de satisfacción. La utilidad del software ha sido evidenciada por varios trabajos analizando el discurso mediático sobre varios temas como la masificación del turismo (Phi, 2019) o la economía colaborativa (Cheng y Edwards, 2019), entre otros.

\section{Aportaciones previsibles del estudio}

A través de la comparación entre el discurso de los medios de comunicación acerca del término "innovación disruptiva" y los temas de investigación relacionados con el concepto examinados por literatura académica se espera contribuir al desarrollo del área del conocimiento y al debate sobre el fenómeno. Por un lado, el análisis del discurso de los medios de comunicación revelará cuáles son los temas que configuran el panorama mediático para así detectar si existen problemáticas relacionadas con los procesos de innovación disruptiva que preocupan a la sociedad. A su vez, los investigadores podrían dar respuesta a estos temas de interés o problemas, abriendo nuevas líneas de investigación académica. Por otra parte, la comparación entre la literatura académica sobre innovación disruptiva y el discurso de los medios de comunicación permitirá detectar si los avances de la investigación han sido diseminados por los medios de comunicación. De esta forma, se puede establecer si existe un cierto alineamiento entre ambas áreas o, por el contrario, los medios deben nutrirse más de la investigación científica cuando redactan sus noticias, y viceversa: los académicos deben estar más atentos a los temas que más interés suscitan en la sociedad e intentar aportar conocimiento y evidencia científica al respecto.

\section{Referencias}

Christensen, C. M. (1997). The innovators' dilemma: When new technologies cause great firms to fail Press. 
Christensen, C. M., Raynor, M. E., \& McDonald, R. (2015). What is disruptive innovation. Harvard Business Review, 93(12), 44-53.

Clark, G. (2003). The disruption opportunity. MIT Sloan Management Review, 44(4), 27.

Djerf-Pierre, M., \& Shehata, A. (2017). Still an agenda setter: Traditional news media and public opinion during the transition from low to high choice media environments. Journal of Communication, 67(5), 733-757.

Dogru, T., Mody, M., \& Suess, C. (2019). Adding evidence to the debate: Quantifying airbnb's disruptive impact on ten key hotel markets. Tourism Management, 72, 27-38.

Falagas, M. E., Pitsouni, E. I., Malietzis, G. A., \& Pappas, G. (2008). Comparison of PubMed, scopus, web of science, and google scholar: Strengths and weaknesses. The FASEB Journal, 22(2), 338-342.

Gómez, P. (2009). Opinión pública y medios de comunicación. teoría de la agenda setting.

Guttentag, D. (2015). Airbnb: Disruptive innovation and the rise of an informal tourism accommodation sector. Current Issues in Tourism, 18(12), 1192-1217.

Hopp, C., Antons, D., Kaminski, J., \& Oliver Salge, T. (2018). Disruptive innovation: Conceptual foundations, empirical evidence, and research opportunities in the digital age. Journal of Product Innovation Management, 35(3), 446-457.

Jensen, K. B. (2002). The complementarity of qualitative and quantitative methodologies in media and communication research. A Handbook of Media and Communication Research: Qualitative and Quantitative Methodologies, 254-272.

King, A. A., \& Baatartogtokh, B. (2015). How useful is the theory of disruptive innovation? MIT Sloan Management Review, 57(1), 77.

Laurell, C., \& Sandström, C. (2018). Comparing coverage of disruptive change in social and traditional media: Evidence from the sharing economy. Technological Forecasting and Social Change, 129, 339-344.

Leung, X. Y., Xue, L., \& Wen, H. (2019). Framing the sharing economy: Toward a sustainable ecosystem. Tourism Management, 71, 44-53.

Li, M., Porter, A. L., \& Suominen, A. (2018). Insights into relationships between disruptive technology/innovation and emerging technology: A bibliometric perspective. Technological Forecasting and Social Change, 129, 285-296.

Markides, C. (2006). Disruptive innovation: In need of better theory. Journal of Product Innovation Management, 23(1), 19-25.

Martin, C. J. (2016). The sharing economy: A pathway to sustainability or a nightmarish form of neoliberal capitalism? Ecological Economics, 121, 149-159.

McCombs, M. (2005). A look at agenda-setting: Past, present and future. Journalism Studies, 6(4), 543-557. 
Petzold, N., Landinez, L., \& Baaken, T. (2019). Disruptive innovation from a process view: A systematic literature review. Creativity and Innovation Management, 28(2), 157-174.

Phi, G. T. (2019). Framing overtourism: A critical news media analysis. Current Issues in Tourism, 15.

Sandström, C., Magnusson, M., \& Jörnmark, J. (2009). Exploring factors influencing incumbents' response to disruptive innovation. Creativity and Innovation Management, 18(1), 8-15.

Smith, A. E., \& Humphreys, M. S. (2006). Evaluation of unsupervised semantic mapping of natural language with leximancer concept mapping. Behavior Research Methods, 38(2), 262-279.

Zhang, W., Daim, T., \& Zhang, Q. (2018). Understanding the disruptive business model innovation of E-business microcredit: a comparative case study in China. Technology Analysis \& Strategic Management, 30(7), 765-777. 